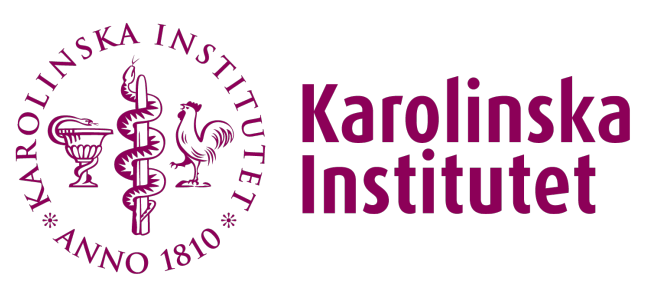

Karolinska Institutet

http://openarchive.ki.se

This is a Peer Reviewed Accepted version of the following article, accepted for publication in Lancet Child and Adolescent Health.

\title{
Efficacy and cost-effectiveness of
}

\section{therapist-guided internet cognitive}

behavioural therapy for paediatric anxiety disorders : a single-centre, single-blind, randomised controlled trial

Jolstedt, Maral; Wahlund, Tove; Lenhard, Fabian; Ljótsson, Brjánn; Mataix-Cols, David; Nord, Martina; Öst, Lars-Göran; Högström, Jens; Serlachius, Eva; Vigerland, Sarah

Lancet Child Adolesc Health. 2018 Nov;2(11):792-801.

http://doi.org/10.1016/S2352-4642(18)30275-X

http://hdl.handle.net/10616/46701

If not otherwise stated by the Publisher's Terms and conditions, the manuscript is deposited under the terms of the Creative Commons Attribution-NonCommercial-NoDerivatives License (http://creativecommons.org/licenses/by-nc-nd/4.0/), which permits non-commercial re-use, distribution, and reproduction in any medium, provided the original work is properly cited, and is not altered, transformed, or built upon in any way. 


\section{(1) \\ Karolinska Institutet}

This is the peer reviewed version of the following article:

Efficacy and cost-effectiveness of therapist-guided internet cognitive behavioural therapy for paediatric anxiety disorders : a single-centre, single-blind, randomised controlled trial.

Jolstedt, Maral; Wahlund, Tove; Lenhard, Fabian; Ljótsson, Brjánn; Mataix-Cols, David; Nord, Martina; Öst, Lars-Göran; Högström, Jens; Serlachius, Eva; Vigerland, Sarah.

The Lancet Child \& Adolescent Health, 2018:2(11), 792801.

which has been published in its final form at:

DOI: http://dx.doi.org/10.1016/S23524642(18)30275-X

This article may be used for non-commercial purposes in accordance with Elsevier Terms and Conditions for selfarchiving.

Publisher: Elsevier 


\section{ACCEPTED MANUSCRIPT}

\section{Efficacy and cost-effectiveness of therapist-guided internet cognitive behaviour therapy for paediatric anxiety disorders: a single-blind randomised controlled trial}

Maral Jolstedt, Tove Wahlund, Fabian Lenhard, Brjánn Ljótsson, David Mataix-Cols, Martina Nord, Lars-Göran Öst, Jens Högström, Eva Serlachius, Sarah Vigerland

Centre for Psychiatry Research, Department of Clinical Neuroscience, Karolinska Institutet, Stockholm, Sweden (M Jolstedt MSc, T Wahlund MSc, F Lenhard PhD, B Ljótsson PhD, Prof D Mataix-Cols, M Nord MSc, J Högström PhD, E Serlachius PhD, S Vigerland PhD); Stockholm Health Care Services; Stockholm County Council, Stockholm, Sweden (M Jolstedt MSc, T Wahlund MSc, F Lenhard PhD, Prof D Mataix-Cols, M Nord MSc, J Högström PhD, E Serlachius PhD, S Vigerland PhD); Department of Clinical Neuroscience, Karolinska Institutet, Stockholm, Sweden (B Ljótsson PhD); Department of Psychology, Stockholm University, Stockholm, Sweden (Prof L G Öst).

Correspondence to: Maral Jolstedt MSc, Centre for Psychiatry Research, Department of Clinical Neuroscience, Karolinska Institutet, \& Stockholm Health Care Services, Stockholm County Council, CAP Research Centre, Gävlegatan 22, SE-113 30 Stockholm, Sweden, maral.jolstedt@ki.se.

\section{Reference to article:}

Jolstedt, M., Wahlund, T., Lenhard, F., Ljótsson, B., Mataix-Cols, D., Nord, M., ... \& Vigerland, S. (2018). Efficacy and cost-effectiveness of therapist-guided internet cognitive behavioural therapy for paediatric anxiety disorders: a single-centre, single-blind, randomised controlled trial. The Lancet Child \& Adolescent Health, 2(11), 792-801.

\section{Link to the article on the Lancet website:}

https://www.sciencedirect.com/science/article/pii/S235246421830275X 


\section{Summary}

Background: Paediatric anxiety disorders are prevalent and associated with substantial disability and long-term adverse consequences. Only a minority of affected children have access to evidence-based treatment; internet-delivered cognitive behaviour therapy (ICBT) could help increase accessibility but needs further rigorous evaluation. The objective of this trial was to evaluate the clinical efficacy and cost-effectiveness of ICBT in the treatment of paediatric anxiety disorders.

Methods: In this single-blind randomised controlled trial, children 8-12 years with a principal anxiety disorder diagnosis were randomly allocated (1:1) to ICBT or internet-delivered child-directed play (ICDP), an active comparator aimed to improve parent-child relationships and increase child self-esteem without directly targeting anxiety. The study was conducted at a single site within the Stockholm specialist child and adolescent mental health services (CAMHS). Primary endpoint was clinician-rated symptom severity (CSR) of the principal anxiety disorder at post-treatment. All participants were included in the primary analysis (intent-to-treat). Trial registration number: NCT02350257.

Findings: Participants $(N=131)$ were recruited between March 2015 and October 2016, mainly via advertisement and referrals from CAMHS, and allocated to ICBT $(n=66)$ or ICDP $(n=65)$. Analyses revealed greater reduction of symptom severity in favour of ICBT (mean difference $0 \cdot 79,95 \%$ CI 0.42 to $1 \cdot 16, p=0 \cdot 002$; Cohen's $d=0 \cdot 77,95 \%$ CI 0.40 to $1 \cdot 15)$ and at post-treatment, $48 \%(n=29)$ of participants randomised to ICBT no longer fulfilled their principal diagnosis, compared to $15 \%(n=9)$ in ICDP (odds ratio $5 \cdot 41,95 \%$ CI $2 \cdot 26$ to $12 \cdot 90, \mathrm{p}<0 \cdot 0001)$. Numbers needed to treat were 3 (95\% CI $2 \cdot 85$ to $3 \cdot 15)$. Treatment gains were maintained at 3-month follow-up, at which point $70 \%(n=40)$ of participants randomised to ICBT no longer met the criteria for their principal anxiety disorder. ICBT resulted in an average societal cost saving of $€ 493.05$ (95\% CI $477 \cdot 17$ to $508 \cdot 92)$ per participant. No severe adverse events were reported.

Interpretation: ICBT is a cost-effective treatment for paediatric anxiety disorders that should be considered for implementation in routine clinical care. 
Funding: The Swedish Research Council for Health, Working Life and Welfare (grant number 20144052) and Stockholm County Council (grant number 14099).

\section{Introduction}

Psychiatric disorders constitute a rising global problem due to high prevalence, great disease burden and increased societal costs. ${ }^{1}$ Anxiety disorders are amongst the most common, with a global worldwide prevalence of around $6 \cdot 5 \%,{ }^{2}$ They often have their onset in childhood, ${ }^{3}$ and can lead to significant disability, reduced quality of life and long-term medical and psychosocial adversity. ${ }^{4}$ Treatment of anxiety disorders early in life is therefore imperative. Cognitive behaviour therapy (CBT) is considered the first-line treatment for paediatric anxiety disorders, ${ }^{5}$ but only a fraction of those who need it have access to this treatment. ${ }^{6}$ Multiple access barriers have been identified, such as geographical distances and limited healthcare resources, and internet-delivered cognitive behaviour therapy (ICBT) has been put forward as a way to potentially increase the availability of evidence-based psychological treatments in a cost-effective manner. ${ }^{7}$ To date, six randomised controlled trials (RCT) have evaluated ICBT with limited online therapist support in school-aged children and adolescents with anxiety disorders, with promising results. ${ }^{8-13}$ However, before ICBT can be recommended for implementation in routine clinical care, more rigorous trials employing active control conditions that reduce the impact of potential confounders are needed. ${ }^{14}$ The main aim of this study was to evaluate the clinical efficacy and costeffectiveness of a therapist-guided ICBT protocol for children with anxiety disorders (BiP Anxiety), ${ }^{8}$ compared to an active control condition (internet-delivered Child-Directed Play; ICDP), which aimed to improve parent-child relationships and increase the child's self-esteem but did not directly target anxiety. It was hypothesized that ICBT would be more efficacious and cost-effective than ICDP. 


\section{Method}

\section{Study design}

The current study was a single blind-, parallel assignment-, randomised-controlled trial comparing 12 weeks of either therapist-supported and parent-assisted ICBT or ICDP. Participants initially randomised to ICDP were offered the option to cross-over to ICBT after the primary end point (post treatment). All participants were naturalistically followed-up three months after ICBT. The study was conducted at a single site, a clinical research unit at the child and adolescent mental health services (CAMHS) in Stockholm, Sweden. This trial also collected long-term follow-up data in a stepped-care design, where non-responders were offered regular face-to-face CBT, which will be presented in a separate paper. The study protocol was approved by the Stockholm Regional Ethical Review Board (reference numbers 2014/1885-31 and 2015/316-31/1).

\section{Participants}

Participants were recruited nationally through newspaper advertisements and through referrals from CAMHS or primary care centres in Sweden. Inclusion criteria were (a) a principal diagnosis of separation anxiety disorder (SEP), generalized anxiety disorder (GAD), specific phobia (SP), social anxiety disorder (SAD) or panic disorder (PD) of at least moderate severity, b) 8-12 years of age, (c) ability to read and write in Swedish, (d) daily access to the internet, (e) a parent or caregiver who could participate in treatment, and (f) if on psychotropic medication, a stable dose for at least six weeks prior to assessment. Exclusion criteria were (a) a diagnosis of autism spectrum disorder, bipolar disorder, psychosis or severe eating disorder (b) current risk of suicide, (c) on-going substance dependence, and (d) completed CBT (>4 sessions of exposure treatment) within the last six months. Caregivers provided written consent to their child's participation in the study. 


\section{Randomisation and masking}

Participants were randomly assigned to either ICBT or ICDP at a 1:1 ratio. Block sizes randomly varying between four and six and were generated using a computer random number generator (www.sealedenvelope.com). An independent researcher was responsible for the generated sequence and the allocation of participants to the two conditions. The allocation was concealed for members of the research group by opaque sealed envelopes. The study-coordinators (MJ and TW) were responsible for enrolment of participants and assigned participants to clinicians. Participants were informed that they would either be allocated to ICBT or a novel online intervention that had not been previously tested (ICDP). Clinicians conducting post-treatment assessments were blinded to the participant's allocation. To measure blinding integrity, all assessors guessed each participant's treatment allocation and recorded whether the participating families had inadvertently revealed their group allocation. ${ }^{15}$ As per protocol, blinding was broken at post treatment (primary endpoint). The naturalistic three-month follow-up assessments were thus unblinded.

\section{Procedures}

All participants were briefly screened via telephone to assess basic inclusion/exclusion criteria. Eligible participants were then scheduled for a face-to-face interview where inclusion/exclusion criteria were further assessed. Assessors conducted face-to-face interviews with participants at post-treatment and three-month follow-up. The ten clinical psychologists involved in the trial conducted all assessments and provided therapist support to participants randomised to both ICBT and ICDP. All psychologists were trained on the principles of ICBT and had weekly supervision. To increase inter-rater reliability on the primary outcome measure, all assessors underwent two days of training. After the training, assessors' ratings had to match those of an expert rater on at least two out of three pre-recorded assessments.

\section{Interventions}

Both ICBT and ICDP are completely web-based treatment programmes with limited weekly asynchronous support from a clinician. The programmes, both comprising 12 modules presented over 
12 weeks, consist of texts, films, illustrations and exercises, and participants are asked to complete one module before having access to the next. The last module is presented at week 12 regardless of how many modules the participant has completed. Therapist contact consists mainly of messages and comments on worksheets within the programme. The therapists are instructed to (1) encourage and support, (2) answer questions, (3) help troubleshooting, (4) clarify the rationale for treatment (if needed), (5) refer back to treatment content, and to 6) prompt and remind participants to log in and work with the program (in case of inactivity).

ICBT was based on the BiP Anxiety ${ }^{8}$ treatment protocol, where parents work together with the child through the various treatment modules. Treatment focuses mainly on exposure therapy, a behavioural intervention involving the gradual confrontation of feared situations and/or stimuli. Parents also access 12 separate parent-directed modules where they learn how parental behaviours can maintain anxiety and how to best support their child through treatment. BiP Anxiety is a generic exposure-based anxiety treatment programme that exists in five disorder-specific versions. The five versions are largely identical but have diagnosis-specific examples and some diagnosis-specific treatment components. Participants are assigned to one of the 5 versions after the initial assessment, depending on their principal anxiety disorder diagnosis. See Supplemental Table 1 for an overview of the BiP Anxiety modules and Supplemental Figure 1 for screenshots from the BiP Anxiety programme.

The active comparator, ICDP, is directed to the parent only, and is based on a commonly used parentdirected intervention for children with conduct problems, which is intended to strengthen the parentchild relationship. ${ }^{16}$ Parents are instructed to spend approximately 20 minutes, 3-4 times per week, playing with their child in a non-directive and praising manner and report back as homework assignments. ICDP was designed to control for therapist attention, for monitoring of symptoms, and for engaging participants in behaviour change (i.e., acquiring and practicing new skills), analogue with core features in ICBT. See Supplemental Table 1 for an overview of the ICDP modules. 


\section{Outcomes}

The primary outcome measure was the blind-rated Clinician Severity Rating (CSR) derived from the Anxiety Disorder Interview Schedule for DSM-IV: parent and child versions (ADIS-C/P) ${ }^{17}$. A CSRrating of $\geq 4$ corresponds to meeting the diagnostic criteria for the principal diagnosis according to the ADIS-C/P.

Secondary outcomes were clinician-assessed, blind-rated global functioning measured with the Children's Global Assessment Scale (CGAS) ${ }^{18}$ and improvement of global functioning measured with the Clinical Global Impression Scale - Improvement (CGI-I). ${ }^{19}$ The CGI-I ranges from $1=$ "Very much improved" to 7 = "Very much worse". Secondary outcome measures also included parent- and childrated anxiety and depression symptoms measured with the Revised Children's Anxiety and Depression Scale (RCADS), ${ }^{20,21}$ everyday functioning measured with the Education, Work and Social Adjustment Scale (EWSAS), ${ }^{22}$ and quality of life measured with KIDSCREEN-10. ${ }^{23}$ RCADS includes anxiety (RCADS-TAS) and major depressive disorder (MDDS) subscales. Only the RCADS-TAS was used in the secondary outcome analyses. The MDDS was used to measure depressive symptoms at pre-treatment only.

Child- and parent reported data were collected through a secure online platform, and clinician-reported data were mainly collected manually on case report forms. See Supplemental Table 2 for detailed information about the timing of measures throughout the study.

Treatment expectancy/credibility was assessed at week 3, after at least one module was completed and families had had a chance to familiarise themselves with the treatment; participants were asked whether they thought that the treatment would be helpful in improving their child's wellbeing (yes/no). Adherence (i.e., treatment compliance) was measured by recording how many modules were completed at the end of treatment. Engagement was measured by asking clinicians whether the participant actively and regularly worked with the exercises presented in the treatment programme (yes/no). Satisfaction was measured post treatment; participants were asked whether they were satisfied with the intervention (yes/no). Therapist time was measured as total time spent supporting the participants throughout the 12- 
week treatment period (time spent online and on the telephone with both child and parent). Additional measures were parental psychiatric symptoms measured with Hospital Anxiety and Depression scale $(\mathrm{HADS})^{24}$ at pre-treatment, and self-reported adverse events at post-treatment. Measures of technology acceptance and specific phobia will be reported separately in additional papers.

Costs associated with healthcare consumption (e.g., physician- and psychologist appointments), supportive resources (e.g. study help), medication, dietary supplements, the child's absence from school, parents' absence from work and productivity losses when in school or at work were measured with the parent-rated Trimbos/iMTA questionnaire for patients with a psychiatric disorder (TiC-P) ${ }^{25}$ at pre- and post treatment. The costs of ICBT and ICDP were estimated using individual per participant therapist times and online platform maintenance costs (i.e., IT support, server costs and software updates).

\section{Statistical analysis}

Power analysis was based on the BiP Anxiety programme showing a between-group effect size (Cohen's $d$ ) of 1.66 when compared to a waitlist condition ${ }^{8}$ and aimed to detect a medium effect $(d=0 \cdot 5)$ when compared to an active control condition. A sample size of $N=128$ ( $N=64$ in each condition) was calculated to be sufficient based on $80 \%$ power with an alpha set at $0 \cdot 05$ (two-tailed).

Linear mixed models (LMM) were used to analyse differences on all continuous primary- and secondary measures between conditions from pre- to post treatment. Piecewise LMM were used for within-group analysis where follow-up data were included in the analysis with one knot at post treatment. Weekly measures were included in the models when available. Unblinded follow-up data were not used for the primary analysis (only pre- to post treatment data were included). Estimates for follow-up, as visually presented in Figure 2, were based on within-group analysis. All available data $(N=131)$ were used and no cases were excluded in the analysis. LMM were created by sequentially adding (a) random intercept, (b) random effect of time, (c) diagonal covariance type for random effects, and/or, (d) unstructured covariance structure for measurements. See Supplemental Table 3 for a description of chosen random effects and covariance structures for all outcome measures. Chi-square tests of model fit were used to evaluate and compare models and the model with the best log likelihood value was chosen before adding 
condition as a covariate. Effect size (Cohen's $d$ ) was based on estimated means derived from the LMM together with the observed pooled standard deviation. ${ }^{26}$ Between-group effect size was based on differences in estimated means at post treatment together with the observed pooled between-group standard deviation at post treatment. Within-group effect size was based on differences in estimated means within a group combined with the observed pooled within-group standard deviation.

Cost data were analysed from a societal perspective, including the direct costs of the two experimental interventions, healthcare costs, and other societal costs. Individual participant resource-use frequencies at pre- and post-treatment time-points were multiplied with their respective unit costs. For a detailed description of cost estimates see Supplemental Table 4. Costs were converted from Swedish krona (SEK) to Euro (€) according to the 2016 conversion rates. The full three-month period between pre- and post-treatment was estimated using linear interpolation, since TIC-P retrospectively covers a period of one month. Missing data for costs were, due to non-normally distributed data, handled with a nonparametric model for imputation based on random forest estimation. ${ }^{27}$ KIDSCREEN-10 scores were converted to quality-adjusted life years (QALYs) using validated mapping algorithms. ${ }^{28}$ Total costs, remission status, and QALYs were, due to the skewed nature of cost data, resampled using nonparametric bootstrapping with 5000 repetitions, and presented as mean costs with CI, mean cost differences between ICBT and ICDP, and incremental cost-effectiveness ratios (ICER; the ratio of costand effect differences between the interventions, indicating the incremental additional costs, or costsavings, for one additional QALY or participant in remission). To test the robustness of the results, sensitivity analyses were carried out by calculating the probability of cost-effectiveness within different willingness-to-pay scenarios (i.e., cost-effectiveness acceptability curve), and by increasing the cost of ICBT with 50\%. Costs were not discounted, as the time frame of the study was less than one year.

Statistical significance was set at $p<0.05$ and $95 \%$ CI were used. The Wald method was used to determine confidence interval for numbers needed to treat (NNT) ${ }^{29}$ Treatment response and remission were defined a priori as at least "Much improved" $(<3)$ on the CGI-I, and as no longer meeting the criteria for the principal anxiety disorder ( $\mathrm{CSR}<4$ on the primary outcome measure), respectively. Normal probability plots, residual plots, and Cook's distance were used to check assumptions for 
parametric tests. Independent $t$-tests were used to analyse patterns of missing data at post treatment and follow-up. Independent $t$-tests, Chi-square tests and Fischer's exact tests were used when comparing the treatment groups. Inter-rater reliability for the primary outcome measure was analysed using intraclass correlation coefficient (ICC). Twenty percent of pre-treatment assessments were randomly selected and re-assessed by an assessor blinded to the original assessment.

SPSS version 23 and R Studio were used for the statistical analysis. The authors of the paper conducted all the statistical analysis. To reduce bias, analyses of treatment efficacy were conducted on all participants and condition was added lastly as a covariate. Resource-use frequencies and their cost estimates were calculated without knowledge of the participants' allocation. Trial registration: NCT02350257 (www.clinicaltrials.gov).

\section{Funding}

The Swedish Research Council for Health, Working Life and Welfare (Forte 2014-4052) and Stockholm County Council (HNSV 14099) funded the study. The funders of the study had no role in study design, data collection, data analysis, data interpretation, or writing of the report. The corresponding author had full access to all data in the study and had the final responsibility for the decision to submit for publication.

\section{Results}

Participants $(N=131)$ were recruited from March 2015 to October 2016 and randomly allocated to ICBT ( $n=66)$ or ICDP ( $n=65)$. Periods of follow-up were from October 2015 to April 2017. For a detailed view of participant flow during the trial, see Figure $\mathbf{1}$ and for a summary of baseline demographic and clinical data, see Table 1. The median travel distance to our clinic was nearly $16 \mathrm{~km}$ (IQR $9 \cdot 4$ to $25 \cdot 5$ ). Ten participants (8\%) dropped-out from the study during treatment and did not provide post-treatment data. These participants had significantly higher parent-reported child anxiety symptoms $(t=2 \cdot 021$, 
$p=0 \cdot 048)$, lower parent-reported child quality of life $(t=2 \cdot 269, p=0 \cdot 027)$ and a higher number of comorbid diagnoses $(t=2 \cdot 678, p=0 \cdot 009)$ at pre-treatment.

Insert Figure 1 and Table 1 approximately here (figure legend at the end of the manuscript)

The inter-rater reliability for the primary outcome measure, CSR, for all anxiety disorders, was good (ICC $0 \cdot 724,95 \%$ CI 0.56 to $0 \cdot 83$ ). See Table 2 for a detailed summary of statistics on all primary- and secondary outcome measures. Participants in both conditions improved significantly from pre- to posttreatment on the primary outcome measure (within-group effect size for ICBT: $d=1 \cdot 22,95 \%$ CI $0 \cdot 78$ to 1.65; for ICDP: $d=0 \cdot 72,95 \%$ CI 0.44 to $1 \cdot 00$ ). However, participants allocated to ICBT improved significantly more than those allocated to ICDP (estimated mean difference $0 \cdot 79,95 \%$ CI 0.42 to $1 \cdot 16$; $p=0 \cdot 002$; Figure 2, left panel). The estimated between-group effect size at post-treatment was $d=0 \cdot 77$, 95\% CI $0 \cdot 40$ to $1 \cdot 15$ ). This was also true for clinician-rated functional impairment, CGAS (estimated mean difference $3 \cdot 96,95 \%$ CI $1 \cdot 14$ to $6 \cdot 78 ; \mathrm{p}=0 \cdot 027 ; d=0 \cdot 51,95 \%$ CI $0 \cdot 14$ to $0 \cdot 88$ ), and parent-rated child anxiety symptoms, RCADS-TAS-P (estimated mean difference $4 \cdot 43,95 \%$ CI $0 \cdot 21$ to $9 \cdot 07$, $p<0 \cdot 0001, d=0 \cdot 35,95 \%$ CI $0 \cdot 02$ to $0 \cdot 72$ ). ICBT participants continued to improve significantly from post-treatment to three-month follow up (estimated mean difference $0 \cdot 64,95 \%$ CI 0.42 to $0 \cdot 87$; $\mathrm{p}<0 \cdot 0001)$. See Supplemental Table 5 for the within-group effects on all primary- and secondary outcomes for the ICBT treatment condition and Supplemental Table 6 for between- and within group effects based on observed data.

Insert Table 2 approximately here

Based on available data, $51 \%(n=30)$ of participants in ICBT were classified as responders $(\mathrm{CGI}-\mathrm{I}<3)$ at post-treatment, compared to $16 \%(n=10)$ in ICDP (odds ratio $5 \cdot 28,95 \%$ CI $2 \cdot 57$ to $12 \cdot 33$, p $<0 \cdot 0001$ ). Additionally, 48\% ( $n=29)$ of participants in ICBT no longer fulfilled diagnostic criteria for their principal anxiety diagnosis $(\mathrm{CSR}<4)$ at post treatment compared to $15 \%(n=9)$ in ICDP (odds ratio 5.41, 95\% CI $2 \cdot 26$ to $12 \cdot 90, \mathrm{p}<0 \cdot 0001)$. Numbers needed to treat $(\mathrm{NNT})$ for ICBT to gain one additional participant in remission (i.e., no longer meeting the criteria for the principal anxiety disorder) were three (95\% CI $2 \cdot 85$ to $3 \cdot 15)$. Seventy percent $(n=40)$ of participants in ICBT no longer met the criteria for 
their principal anxiety disorder at follow-up. See Supplemental Table 7 for a summary of response and remission data.

Fifty-seven participants crossed over to ICBT after completed ICDP (primary end-point), and six of these no longer fulfilled diagnostic criteria for their principal anxiety disorder before starting ICBT treatment. Intent-to-treat analysis show that participants improved significantly on the primary outcome measure, CSR, from immediately before to after ICBT (estimated mean difference $1 \cdot 73,95 \%$ CI $0 \cdot 83$ to $1 \cdot 27, \mathrm{p}<0 \cdot 0001$ ) and continued to improve further three months after ICBT (estimated mean difference $0 \cdot 41,95 \%$ CI $0 \cdot 17$ to $0 \cdot 64, \mathrm{p}=0 \cdot 001$; Figure 2, right panel and Supplemental Table 8). Based on available data and only those who fulfilled diagnostic criteria for their principal anxiety disorder before crossing over to ICBT $(n=46), 52 \%(n=24)$ were in remission after ICBT, and $60 \%$ $(n=28)$ were in remission three months after ICBT. The mean number of completed ICBT modules were $M=6 \cdot 47(S D=3 \cdot 46)$.

Insert Figure 2 approximately here (figure legend at the end of the manuscript)

Treatment condition was inadvertently revealed to blind assessors in $12 \%$ of cases ( $n=12$ in ICBT and $n=2$ in ICDP). Post-hoc analyses excluding these participants confirmed the superiority of ICBT over ICDP (estimated mean difference $0 \cdot 53,95 \%$ CI $0 \cdot 12$ to $0 \cdot 87 ; p=0 \cdot 010 ;$ Supplemental Table 9), though the estimated between-group effect size at post-treatment was smaller $(d=0 \cdot 49,95 \%$ CI $0 \cdot 12$ to $0 \cdot 87)$. The proportions of participants classified as responders and in remission at post-treatment were similar to those of the main analysis (see Supplemental Table $\mathbf{1 0}$ for a summary of response and remission data in this subgroup).

Data on treatment credibility (week 3), adherence, engagement and satisfaction, together with therapist support time are presented in Table 3. ICBT was rated as significantly more credible than ICDP $\left(x^{2}\right.$ 9.60, $p=0 \cdot 002)$ three weeks into treatment. However, post-hoc analyses showed that treatment credibility was not significantly associated with treatment response, remission, adherence or engagement in treatment, or with improvement on the primary outcome measure (Supplemental Tables 11-12 and Supplemental Figure 2). The number of completed modules was similar across the two 
groups. For example, 92\% $(n=55)$ in the ICBT, and $80 \%(n=47)$ in the ICDP groups completed at least four modules, meaning they had started to actively work with the key components of the treatment. Clinicians rated their participants as being equally engaged in both treatments. Clinicians spent more time supporting participants in the ICBT than in the ICDP groups. Families randomised to ICBT had higher satisfaction ratings at post-treatment.

Insert Table 3 approximately here

Cost-effectiveness analyses showed that the total societal costs were almost $€ 500$ lower in the ICBT condition compared to ICDP at post treatment (mean difference $€-493 \cdot 05,95 \%$ CI $-508 \cdot 92$ to $-477 \cdot 17$ ). Reduced healthcare costs were the largest component of the total cost difference (mean difference $€ 348 \cdot 89,95 \%$ CI $337 \cdot 39$ to $360 \cdot 38$; Supplemental Figure 3). For a summary of resource use see Supplemental Table 13. ICBT was associated with cost savings in all cost categories except for therapist time, where costs were on average $€ 83.03$ higher, where the cost of ICBT was on average $€ 158.89$ (95\% CI $140 \cdot 50$ to $177 \cdot 28$ ), and the cost of ICDP $€ 75.69$ (95\% CI $68 \cdot 58$ to $82 \cdot 79$ ). The incremental cost-effectiveness ratio (ICER) regarding total costs and remission was $€-1436 \cdot 81$ (95\% CI $-1493 \cdot 36$ to $-1380 \cdot 26)$, indicating cost savings and higher probability of remission in ICBT compared to ICDP. Only minimal differences regarding QALYs were observed (mean difference of 0.02), due to which the calculation of an ICER value was not meaningful. Figure 3 depicts the corresponding costeffectiveness planes, i.e., the probabilistic distributions of cost and effect differences between the two interventions with values in the south-east quadrant indicating that ICBT is cost saving and more effective than ICDP. Overall, the probability of ICBT being cost effective was approximately $80 \%$ in a scenario where the willingness to pay more for the additional effect of ICBT would be $€ 0$, and approximately $100 \%$ at a willingness to pay $€ 5000$. See Figure 4 for the cost-effectiveness acceptability curve displaying the probability of ICBT of being cost-effective over ICDP given different willingnessto-pay scenarios. Sensitivity analyses showed that increasing the cost of ICBT with 50\% decreased the cost saving effect of ICBT over ICDP only marginally to $€-473.53$ (95\% CI $-491 \cdot 47$ to $-455 \cdot 59)$.

Insert Figure 3 and 4 approximately here (figure legend at the end of the manuscript) 
No severe adverse events (AE) were reported in either condition. The number of self-reported AE did not differ significantly between treatment conditions $\left(x^{2} 0 \cdot 07, p=0 \cdot 786\right)$. See Table 4 for a detailed overview of reported $\mathrm{AE}$ in each treatment condition.

\section{Discussion}

This is the largest RCT to evaluate the efficacy of therapist-guided ICBT for paediatric anxiety disorders compared to an active control condition, and the first to include a health economic evaluation. A 12week ICBT programme with limited online therapist support was more effective than an internetdelivered control intervention controlling for e.g., mode of delivery, therapist contact, and general behaviour change. This was true for clinician-rated symptom severity and functional impairment, remission rates, and parent-rated child anxiety. At post-treatment, 48\% ( $n=29)$ of participants randomised to ICBT no longer fulfilled their principal diagnosis, compared to $15 \%(n=9)$ in ICDP. Treatment gains were maintained for at least 3 months after the end of treatment, at which point $70 \%$ $(n=40)$ of participants randomised to ICBT no longer met the criteria for their principal anxiety disorder. Overall, these results regarding remission are comparable to previous waitlist-controlled trials of ICBT for paediatric anxiety disorders, ${ }^{8,11}$ and even face-to-face CBT trials for anxiety disorders. ${ }^{5}$ Participants who crossed over to ICBT after the control treatment, also improved robustly on primary and secondary outcome measures. A majority of participants were satisfied with the treatment despite minimal contact with a therapist. No serious AE were reported. ICBT was also more cost-effective than ICDP, resulting in an average societal cost saving of $€ 493$ per participant. The main cost saving factor was the reduced use of healthcare in the ICBT group. Overall, the probability of ICBT being cost effective was high, even in scenarios where the willingness to pay more for ICBT would be zero. Overall, the results indicate that ICBT has potential to greatly increase access to evidence-based treatment for children with anxiety disorders in a cost-effective way.

Interestingly, ICBT was cost-effective compared to ICDP regarding remission rates but not QALYs. The same observation was made in another trial of ICBT for adolescents with obsessive-compulsive disorder. ${ }^{30}$ The changes in QALYs are based on child-reported quality of life, which did not improve in 
either the ICBT or the ICDP conditions. As it is highly unlikely that clinical improvements are not associated with improvements in quality of life, one possible explanation for this could be that available measures of quality of life may lack validity or sensitivity to change in children and adolescents with anxiety disorders.

This represents the largest RCT of ICBT for paediatric anxiety disorders to date and the first to be sufficiently powered to detect a superiority effect over an active control intervention. The inclusion of a truly active control condition helped reduce biases related to therapist support, symptom monitoring, homework assignments and behaviour change. Other strengths included the use and careful verification of the blinding procedures (true double blind trials are not possible in psychological interventions) and minimal data loss. Despite our efforts to present the active control condition as credible and meaningful, it was still rated as being less credible at week 3, which may have affected the expectations for improvement. However, credibility at week 3 did not seem related to either adherence with the treatment (number of completed modules), therapist-rated engagement with the therapy or, crucially, treatment outcomes; participants improved more with ICBT than with ICDP, regardless of whether they thought the treatment was credible or not. Some families inadvertently revealed their group allocation to the blind rater; post-hoc analyses revealed that excluding these participants did not change the overall conclusions of our study but it did reduce the magnitude of the effect sizes. Another potential limitation of the study is that the results may not generalise to the entire population of patients with anxiety disorders because the majority of participants were self-referred and their families highly educated. Thus, despite the fact that many participants had multiple psychiatric comorbidities and a history of previous contact with CAMHS, participants in this trial may have been less complex and particularly motivated to engage in psychological treatment, compared to patients typically seen in regular CAMHS services. Furthermore, participants with missing data were more severely ill at baseline (i.e., higher anxiety, lower quality of life, higher number of comorbid diagnosis), thus potentially limiting the generalizability of our findings to mild to moderately ill children. Also, there was a higher percentage of participants with SAD in ICDP compared to ICBT, which could have affected outcome since evidence suggest that $\mathrm{SAD}$ is associated with poorer treatment outcome in generic CBT. ${ }^{31}$ Future 
research should focus on a better understanding of which participants benefit from ICBT and which do not. Post-hoc sub-analyses of e.g., severity and diagnosis as potential predictors of treatment outcome are planned for a secondary paper deriving from this dataset. Another limitation of the study is that the long-term effects of ICBT are unknown. Future studies with longer controlled follow-ups would be needed to address this question but there are few reasons to believe that ICBT should be different from traditional face-to-face CBT, which has a reasonably well-established durability.

ICBT should not be seen as a substitute for traditional face-to-face CBT but instead as an alternative that may greatly increase access to evidence based treatments for children with anxiety disorders. ICBT is clearly not suitable for the most complex anxiety disorder cases; rather, ICBT may be particularly useful in a stepped-care model, where mild to moderate cases with anxiety disorders can be offered ICBT as first-line treatment, delivered by therapists who are not necessarily experts in anxiety disorders, thus freeing resources for more severe and complex cases. The average therapist support time for ICBT was around 25 minutes per participant per week (including time spent on both child and parent), which is approximately $1 / 3$ of the time required in standard face-to-face CBT. ICBT also has the potential to eliminate logistic barriers, as families do not need to travel to a clinic to receive treatment. ICBT may be particularly promising in rural or sparsely populated areas where access to trained CBT therapists is limited. Future stepped-care trials for children and adolescents with anxiety disorders are warranted.

In conclusion, ICBT is an efficacious and cost-effective treatment for young people with anxiety disorders and its implementation in routine clinical care should contribute to reducing the current gap between demand and availability of evidence-based interventions. 


\section{Contributors}

MJ, BL, DMC, LGÖ, JH, ES and SV contributed to the conception and design of the trial. MJ, FL, BL, DMC and SV contributed to the data analysis of the trial. MJ, TW, MN, JH and SV contributed to the acquisition of data. All authors contributed to drafting the work and revising it critically for important intellectual content as well as giving final approval to the version of the paper being published. All authors who qualify for authorship for this manuscript are included in the authorship.

\section{Declaration of interest}

MJ, ES and SV are the developers of the BiP Anxiety treatment protocol, and MJ, JH and SV are the developers of the active control condition, internet-delivered child-directed play.

\section{Acknowledgements}

The authors would like to acknowledge the contribution of the clinical psychologists working in this trial for assisting with assessment and treatment of participants. The authors would also like to acknowledge Ulrika Thulin, $\mathrm{PhD}$, who helped develop the BiP Anxiety programme.

\section{Data sharing statement}

Trial data are not publicly available owing to data privacy issues, but access to the anonymised dataset, together with the trial protocol and other related documents can be obtained from the corresponding author on reasonable request. 


\section{References}

1. Whiteford HA, Degenhardt L, Rehm J, Baxter AJ, Ferrari AJ, Erskine HE, et al. Global burden of disease attributable to mental and substance use disorders: findings from the Global Burden of Disease Study 2010. The Lancet 2013; 382(9904): 1575-86.

2. Polanczyk GV, Salum GA, Sugaya LS, Caye A, Rohde LA. Annual Research Review: A metaanalysis of the worldwide prevalence of mental disorders in children and adolescents. Journal of Child Psychology and Psychiatry 2015; 56(3): 345-65.

3. Kessler RC, Berglund P, Demler O, Jin R, Merikangas KR, Walters EE. Lifetime prevalence and age-of-onset distributions of DSM-IV disorders in the National Comorbidity Survey Replication. Archives of General Psychiatry 2005; 62(6): 593-602.

4. Bayer JK, Rapee RM, Hiscock H, Ukoumunne OC, Mihalopoulos C, Wake M. Translational research to prevent internalizing problems early in childhood. Depression and Anxiety 2011; 28(1): $50-7$.

5. James AC, James G, Cowdrey FA, Soler A, Choke A. Cognitive behavioural therapy for anxiety disorders in children and adolescents. The Cochrane Library 2015; 2: CD004690.

6. Kazdin AE. Addressing the treatment gap: A key challenge for extending evidence-based psychosocial interventions. Behaviour Research and Therapy 2017; 88: 7-18.

7. Holmes EA, Ghaderi A, Harmer CJ, Ramchandani PG, Cuijpers P, Morrison AP, et al. The Lancet Psychiatry Commission on psychological treatments research in tomorrow's science. The Lancet Psychiatry 2018; 5(3): 237-86.

8. Vigerland S, Ljótsson B, Thulin U, Öst L-G, Andersson G, Serlachius E. Internet-delivered cognitive behavioural therapy for children with anxiety disorders: A randomised controlled trial. Behaviour Research and Therapy 2016; 76: 47-56.

9. Wuthrich VM, Rapee RM, Cunningham MJ, Lyneham HJ, Hudson JL, \& Schniering CA. A randomized controlled trial of the Cool Teens CD-ROM computerized program for adolescent anxiety. Journal of American Academy Child and Adolescent Psychiatry 2012; 51(3), 261-270.

10. Spence SH, Donovan CL, March S, Gamble A, Anderson RE, Prosser S, et al. A randomized controlled trial of online versus clinic-based CBT for adolescent anxiety. Journal of Consulting and Clinical Psychology 2011; 79(5): 629-42. 
11. March S, Spence SH, Donovan CL. The efficacy of an internet-based cognitive-behavioral therapy intervention for child anxiety disorders. Journal of Pediatric Psychology 2009; 34(5): 474-87.

12. Spence SH, Donovan CL, March S, Kenardy JA, Hearn CS. Generic versus disorder specific cognitive behavior therapy for social anxiety disorder in youth: A randomized controlled trial using internet delivery. Behaviour Research and Therapy 2017; 90: 41-57.

13. Tillfors M, Andersson G, Ekselius L, Furmark T, Lewenhaupt S, Karlsson A, et al. A randomized trial of Internet-delivered treatment for social anxiety disorder in high school students. Cognitive Behaviour Therapy 2011; 40(2): 147-57.

14. Vigerland S, Lenhard F, Bonnert M, Lalouni M, Hedman E, Ahlen J, et al. Internet-delivered cognitive behavior therapy for children and adolescents: A systematic review and meta-analysis. Clinical Psychology Review 2016; 50: 1-10.

15. Basoglu M, Marks I, Livanou M, Swinson R. Double-blindness procedures, rater blindness, and ratings of outcome: observations from a controlled trial. Archives of General Psychiatry 1997; 54(8): $744-8$.

16. Kaminsky J, Valle L. Filene, JH \& Boyle, CL. A Meta-analytic review of components associated with parent training program effectiveness. Journal of Abnormal Child Psychology 2008;36(4): 56789.

17. Silverman WK, Albano AM. Anxiety Disorders Interview Schedule for DSM-IV: Parent interview schedule. Albany, NY: Greywind Publications, 1996.

18. Shaffer D, Gould MS, Brasic J, Ambrosini P, Fisher P, Bird H, et al. A children's global assessment scale (CGAS). Archives of General Psychiatry 1983; 40(11): 1228-31.

19. Guy W. Assessment manual for psychopharmacology, revised. Washington DC: US Government Printing Office, 1976.

20. Chorpita BF, Yim L, Moffitt C, Umemoto LA, Francis SE. Assessment of symptoms of DSM-IV anxiety and depression in children: A revised child anxiety and depression scale. Behaviour Research and Therapy 2000; 38(8): 835-55.

21. Spence SH. A measure of anxiety symptoms among children. Behaviour Research and Therapy 1998; 36(5): 545-66.

22. Mundt JC, Marks IM, Shear MK, Greist JH. The Work and Social Adjustment Scale: a simple measure of impairment in functioning. The British Journal of Psychiatry 2002; 180(5): 461-4. 
23. Ravens-Sieberer U, Erhart M, Rajmil L, et al. Reliability, construct and criterion validity of the KIDSCREEN-10 score: a short measure for children and adolescents' well-being and health-related quality of life. Quality of Life Research 2010; 19(10): 1487-500.

24. Zigmond AS, Snaith RP. The hospital anxiety and depression scale. Acta Psychiatrica Scandinavica 1983; 67(6): 361-70.

25. Bouwmans C, De Jong K, Timman R, Zijlstra-Vlasveld M, Van der Feltz-Cornelis C, Tan SS, et al. Feasibility, reliability and validity of a questionnaire on healthcare consumption and productivity loss in patients with a psychiatric disorder (TiC-P). BMC Health Services Research 2013; 13(1): 217.

26. Feingold A. Effect sizes for growth-modeling analysis for controlled clinical trials in the same metric as for classical analysis. Psychological Methods 2009; 14(1): 43-53.

27. Stekhoven DJ, Bühlmann P. MissForest - non-parametric missing value imputation for mixed-type data. Bioinformatics 2011; 28(1): 112-8.

28. Chen G, Stevens K, Rowen D, Ratcliffe J. From KIDSCREEN-10 to CHU9D: creating a unique mapping algorithm for application in economic evaluation. Health and Quality of Life Outcomes 2014; 12(1): 134 .

29. Bender R. Calculating confidence intervals for the number needed to treat. Controlled Clinical Trials 2001; 22(2): 102-10.

30. Lenhard F, Ssegonja R, Andersson E, Feldman I, Rück C, Mataix-Cols D, et al. Cost-effectiveness of therapist-guided internet-delivered cognitive behaviour therapy for paediatric obsessive-compulsive disorder: results from a randomized controlled trial. BMJ Open 2017; 7(5): e015246.

31. Hudson JL, Keers R, Roberts S, Coleman J RI, Breen G, Arendt K, et al. Clinical predictors of response to cognitive-behavioral therapy in pediatric anxiety disorders: the Genes for Treatment (GxT) study. Journal of the American Academy of Child \& Adolescent Psychiatry 2015; 54(6): 454-463. 
Table 1: Pre-treatment demographic and clinical information

\begin{tabular}{|c|c|c|c|}
\hline & Total $(N=131)$ & ICBT $(n=66)$ & ICDP $(n=65)$ \\
\hline Age, $M$ (SD) & $9 \cdot 95(1 \cdot 33)$ & $9.97(1.39)$ & $9 \cdot 92(1 \cdot 28)$ \\
\hline Females, $n(\%)$ & $70(53)$ & $28(42)$ & $42(65)$ \\
\hline Age parent, $M$ (SD) & $42 \cdot 47(4 \cdot 67)$ & $43 \cdot 21(4 \cdot 64)$ & $41 \cdot 71(4 \cdot 28)$ \\
\hline $\begin{array}{l}\text { Educational level parent, } \boldsymbol{n}(\%) \\
<12 \text { years } \\
12 \text { years } \\
\text { University studies } \\
\text { University diploma } \\
\text { Post graduate degree }\end{array}$ & $\begin{aligned} 7 & (5) \\
19 & (15) \\
20 & (15) \\
81 & (62) \\
4 & (3)\end{aligned}$ & $\begin{array}{c}3(5) \\
7(11) \\
11(17) \\
43(65) \\
2(3)\end{array}$ & $\begin{array}{c}4(6) \\
12(18) \\
9(14) \\
38(58) \\
2(3)\end{array}$ \\
\hline HAD parent, $M$ (SD) & $9 \cdot 11(6 \cdot 99)$ & $8 \cdot 74(7 \cdot 32)$ & $9 \cdot 48(6 \cdot 68)$ \\
\hline $\begin{array}{l}\text { Distance to clinic, } \mathrm{km} \\
\quad \text { Min-Max } \\
\text { Median (IQR) }\end{array}$ & $\begin{array}{c}1 \cdot 4-506 \cdot 0 \\
15 \cdot 8(9 \cdot 4-25 \cdot 5)\end{array}$ & $\begin{array}{c}1 \cdot 8-506 \\
15 \cdot 3(9 \cdot 3-24 \cdot 2)\end{array}$ & $\begin{array}{c}1 \cdot 4-483 \cdot 0 \\
17 \cdot 2(9 \cdot 6-26 \cdot 9)\end{array}$ \\
\hline Previous contact with CAMHS, $n(\%)$ & $65(50)$ & $36(55)$ & $29(45)$ \\
\hline Referred from CAMHS or GP, $n(\%)$ & $15(11 \cdot 45)$ & $8(12 \cdot 30)$ & $7(10 \cdot 60)$ \\
\hline Ongoing medication, $n(\%)$ & $13(10)$ & $5(8)$ & $8(12)$ \\
\hline $\begin{array}{l}\text { Principal diagnosis, } \boldsymbol{n}(\%) \\
\text { SEP } \\
\text { GAD } \\
\text { SAD } \\
\text { SP } \\
\text { PD }\end{array}$ & $\begin{array}{l}49(37) \\
27(21) \\
25(19) \\
21(16) \\
9(7)\end{array}$ & $\begin{array}{l}28(42) \\
16(24) \\
7(11) \\
11(17) \\
4(6)\end{array}$ & $\begin{array}{l}21(32) \\
11(17) \\
18(28) \\
10(18) \\
5(8)\end{array}$ \\
\hline $\begin{array}{l}\text { Number of diagnoses, } n(\%) \\
\quad 1 \\
2 \\
3 \\
>3\end{array}$ & $\begin{array}{l}64(49) \\
41(31) \\
16(12) \\
10(8)\end{array}$ & $\begin{array}{c}36(55) \\
20(30) \\
6(9) \\
4(6)\end{array}$ & $\begin{array}{c}28(43) \\
21(32) \\
10(15) \\
6(9)\end{array}$ \\
\hline Depressive symptoms, $M$ (SD) & $7 \cdot 64(3 \cdot 66)$ & $7 \cdot 17(3 \cdot 90)$ & $8 \cdot 12(3 \cdot 36)$ \\
\hline $\begin{array}{l}\text { Abbreviation. } \mathrm{ICBT}=\text { Internet-delivered } \mathrm{C} \\
\mathrm{HAD}=\text { Hospital and Anxiety Scale; } \mathrm{IQR}= \\
\mathrm{GP}=\text { general practioner; } \mathrm{SEP}=\text { Separation } \\
\mathrm{SP}=\text { Specific Phobia; } \mathrm{PD}=\text { Panic Disorder. } \\
\text { the Revised Children's Anxiety and Depre }\end{array}$ & $\begin{array}{l}\text { iour Therapy; ICI } \\
\text { ange; CAMHS }=\mathrm{C} \\
\text { ler; } \mathrm{GAD}=\mathrm{General} \\
\text { ive symptoms mea }\end{array}$ & $\begin{array}{l}\text { net-delivered Chilc } \\
\text { Adolescent Mental } \\
\text { xiety Disorder; SAI } \\
\text { ith the Major depre }\end{array}$ & $\begin{array}{l}\text { ted Play; } \\
\text { h Services; } \\
\text { ial Anxiety Disorder; } \\
\text { disorder subscale in }\end{array}$ \\
\hline
\end{tabular}




\begin{tabular}{|c|c|c|c|c|c|c|c|c|c|c|}
\hline & \multicolumn{4}{|c|}{ Observed $M(S D)$} & \multicolumn{3}{|c|}{ Linear mixed model } & \multicolumn{3}{|c|}{ Effect size (95\% CI) } \\
\hline & $\mathbf{n}$ & ICBT & $\bar{n}$ & ICDP & $\begin{array}{c}\text { Estimated mean } \\
\text { ICBT }\end{array}$ & $\begin{array}{c}\text { Estimated mean } \\
\text { ICDP }\end{array}$ & $p$-value & Between group & $\begin{array}{c}\text { Within group } \\
\text { ICBT }\end{array}$ & $\begin{array}{c}\text { Within group } \\
\text { ICDP }\end{array}$ \\
\hline \multicolumn{11}{|l|}{ CSR } \\
\hline Pre & 66 & $4.73(0 \cdot 73)$ & 65 & $4.92(0 \cdot 76)$ & 4.72 & 4.92 & & & & \\
\hline Post & 60 & $3 \cdot 55(1 \cdot 14)$ & 61 & $4.31(0 \cdot 89)$ & $3 \cdot 54$ & $4 \cdot 33$ & $0 \cdot 002$ & $0.77(0.40$ to 1.15$)$ & $1.22(0.78$ to 1.65$)$ & $0.72(0.44$ to 1.00$)$ \\
\hline \multicolumn{11}{|l|}{ CGAS } \\
\hline Pre & 66 & $57 \cdot 18(5 \cdot 98)$ & 65 & $55.97(5.88)$ & $57 \cdot 18$ & 55.97 & & & & \\
\hline Post & 59 & $63 \cdot 14(7 \cdot 97)$ & 61 & $58.97(7 \cdot 61)$ & $62 \cdot 92$ & 58.96 & $0 \cdot 027$ & $0.51(0.14$ to 0.88$)$ & $0.80(0.47$ to 1.14$)$ & $0.42(0.22$ to 0.63$)$ \\
\hline \multicolumn{11}{|c|}{ RCADS-C } \\
\hline Pre & 66 & $34.67(15 \cdot 38)$ & 65 & $35.00(14 \cdot 38)$ & $34 \cdot 60$ & 34.03 & & & & \\
\hline Post & 54 & $26 \cdot 41(14.42)$ & 56 & $27.50(15.37)$ & $26 \cdot 00$ & $28 \cdot 43$ & $0 \cdot 163$ & $0 \cdot 16(-0 \cdot 22$ to $0 \cdot 54)$ & $0.58(0.33$ to 0.82$)$ & $0 \cdot 38(0 \cdot 16$ to $0 \cdot 59)$ \\
\hline \multicolumn{11}{|c|}{ RCADS-P } \\
\hline Pre & 66 & $39.46(12.53)$ & 65 & $37.65(13 \cdot 17)$ & $39 \cdot 79$ & $37 \cdot 10$ & & & & \\
\hline Post & 56 & $26.25(10 \cdot 75)$ & 60 & $30.98(14.13)$ & $26 \cdot 67$ & $31 \cdot 10$ & $<0 \cdot 0001$ & $0.35(-0.02$ to 0.72$)$ & $1.12(0.79$ to 1.44$)$ & $0.44(0.19$ to 0.68$)$ \\
\hline \multicolumn{11}{|c|}{ EWSAS-C } \\
\hline Pre & 66 & $12 \cdot 70(7 \cdot 86)$ & 65 & $12 \cdot 67(6 \cdot 45)$ & $12 \cdot 72$ & $12 \cdot 22$ & & & & \\
\hline Post & 54 & $8 \cdot 30(6 \cdot 08)$ & 56 & $10 \cdot 25(7 \cdot 31)$ & $8 \cdot 22$ & 9.94 & $0 \cdot 087$ & $0 \cdot 26(-0 \cdot 12$ to 0.63$)$ & $0.63(0.32$ to .95$)$ & $0.33(0.07$ to 0.59$)$ \\
\hline \multicolumn{11}{|c|}{ EWSAS-P } \\
\hline Pre & 66 & $17 \cdot 00(7 \cdot 37)$ & 65 & $17 \cdot 68(7 \cdot 37)$ & $16 \cdot 72$ & $17 \cdot 40$ & & & & \\
\hline Post & 56 & $11 \cdot 41(7 \cdot 27)$ & 59 & $14 \cdot 32(8 \cdot 56)$ & $11 \cdot 08$ & $14 \cdot 22$ & $0 \cdot 095$ & $0.39(0.02$ to 0.77$)$ & $0.77(0.45$ to 1.09$)$ & $0.40(0 \cdot 12$ to $0 \cdot 68)$ \\
\hline \multicolumn{11}{|c|}{ KIDSCREEN-C } \\
\hline Pre & 66 & $39.47(4 \cdot 87)$ & 65 & $37 \cdot 80(4 \cdot 21)$ & $39 \cdot 47$ & $39 \cdot 54$ & & & & \\
\hline Post & 54 & $39.98(5 \cdot 13)$ & 55 & $39 \cdot 67(4 \cdot 60)$ & 37.93 & $39 \cdot 67$ & $0 \cdot 087$ & $-0 \cdot 36(-0.74$ to $0 \cdot 07)$ & $-0 \cdot 31(-0.03$ to $-0 \cdot 58)$ & $0.03(-0.26$ to $0 \cdot 32)$ \\
\hline \multicolumn{11}{|c|}{ KIDSCREEN-P } \\
\hline Pre & 66 & $36 \cdot 45(4 \cdot 10)$ & 65 & $35 \cdot 18(4 \cdot 16)$ & $36 \cdot 45$ & $35 \cdot 18$ & & & & \\
\hline Post & 56 & $37.68(3.89)$ & 59 & $36 \cdot 41(4 \cdot 18)$ & $37 \cdot 36$ & $36 \cdot 19$ & $0 \cdot 881$ & $0.29(-0.08$ to 0.66$)$ & $0.23(-0.02$ to 0.47$)$ & $0.24(0.02$ to 0.46$)$ \\
\hline
\end{tabular}


Table 3: Credibility and treatment adherence

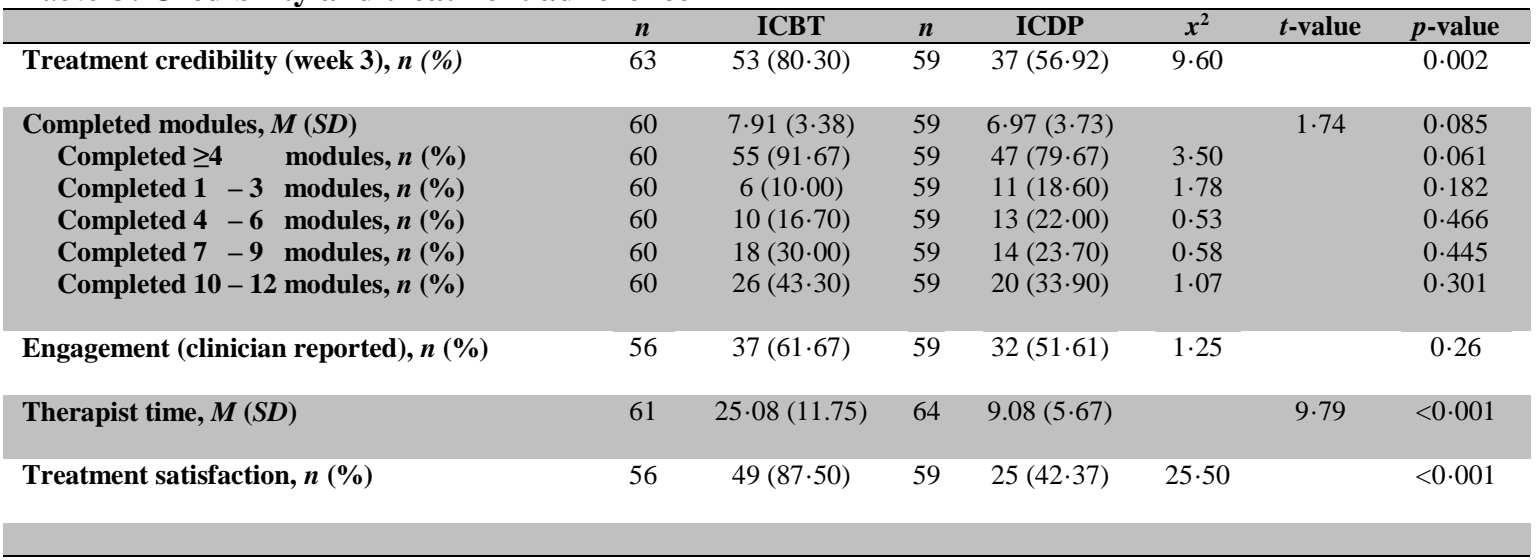

Abbreviations. ICBT=Internet-delivered Cognitive Behaviour Therapy; ICDP=Internet-delivered Child-directed Play. Note.

Treatment time for ICBT includes therapist time spent on both child and parent whereas treatment time for ICDP includes time spent on parent only; Therapist time includes time spent online as well as via telephone. 
Table 4: Adverse events (self-reported) by study group

\begin{tabular}{|c|c|c|c|c|}
\hline & ICBT $(n=54)$ & ICDP $(n=55)$ & $x^{2}$ & $p$-value \\
\hline Depressive symptoms, $n(\%)$ & $2(3 \cdot 7)$ & $2(3 \cdot 6)$ & $0 \cdot 00$ & $0 \cdot 948$ \\
\hline Anxiety symptoms, $n(\%)$ & $9(16 \cdot 7)$ & $13(23 \cdot 6)$ & $2 \cdot 97$ & $0 \cdot 085$ \\
\hline Anger/tantrums, $n(\%)$ & $3(5 \cdot 6)$ & $1(1 \cdot 8)$ & $*$ & $0 \cdot 601$ \\
\hline $\begin{array}{l}\text { Somatic symptoms (e.g., stomach- or head ache, } \\
\text { weight gain), } n(\%)\end{array}$ & $3(5 \cdot 6)$ & None & $*$ & $0 \cdot 227$ \\
\hline $\begin{array}{l}\text { Total self-reported adverse events, } n(\%) \\
\text { Impact at the time of the event, } n(\%) \\
\text { Still an impact at post treatment, } n(\%)\end{array}$ & $\begin{aligned} 17 & (25 \cdot 8) \\
6 & (9 \cdot 1) \\
1 & (1 \cdot 5)\end{aligned}$ & $\begin{array}{c}16(24 \cdot 6) \\
11(16 \cdot 9) \\
6(9 \cdot 2)\end{array}$ & $\begin{array}{l}0 \cdot 07 \\
3 \cdot 69 \\
*\end{array}$ & $\begin{array}{l}0 \cdot 786 \\
0 \cdot 055 \\
0 \cdot 304\end{array}$ \\
\hline
\end{tabular}

Abbreviation. ICBT=Internet-delivered Cognitive Behaviour Therapy; ICDP=Internet-delivered Child-directed Play. Note. *Fischer exact test due to $\leq 5$ frequency. 


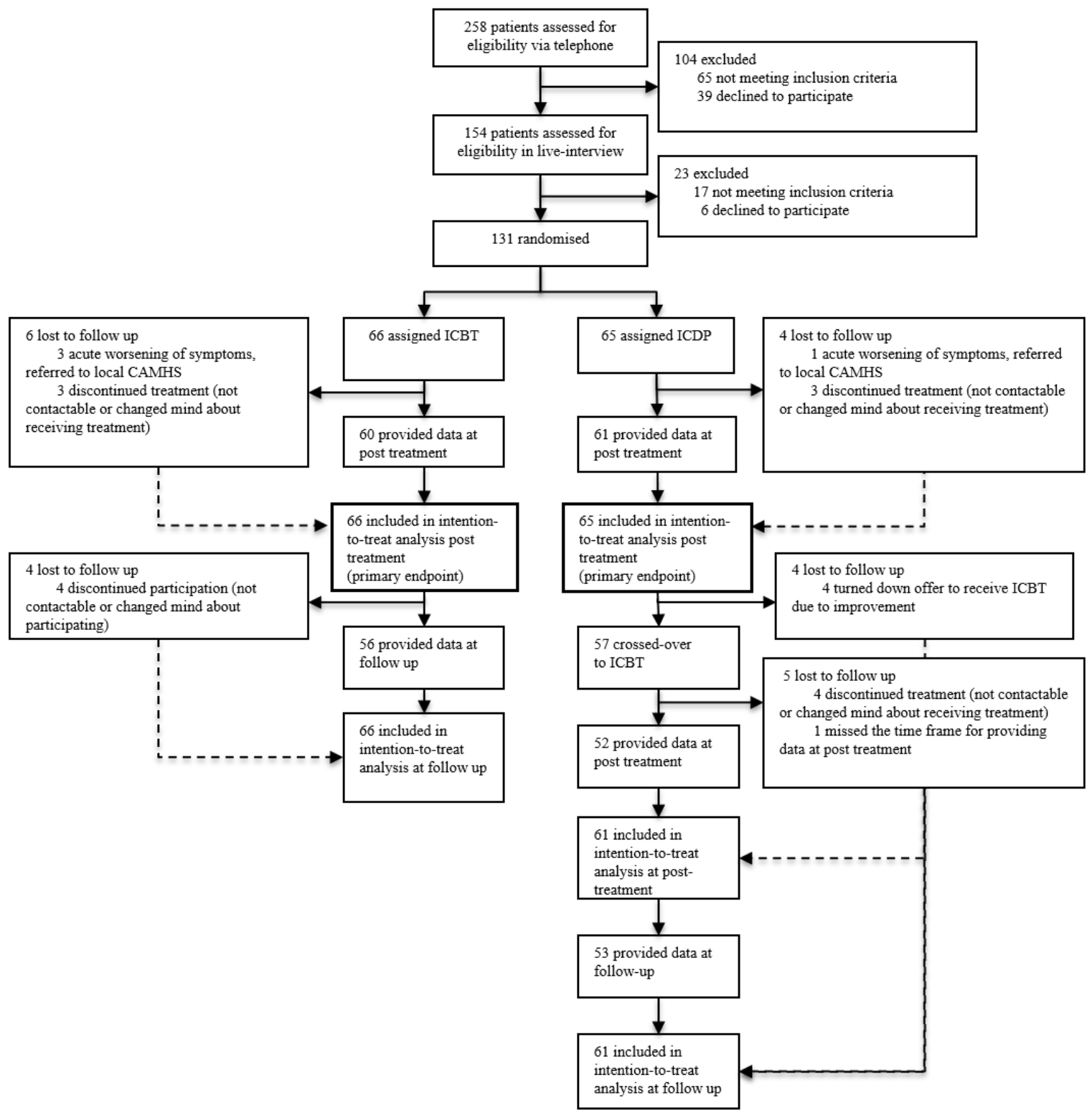

Figure 1: Flow diagram of the progress through the phases of the trial

Abbreviations. ICBT=Internet-delivered Cognitive Behaviour Therapy; ICDP=Internet-delivered Child-directed Play; CAMHS=Child- and Adolescent Mental Health Services. 

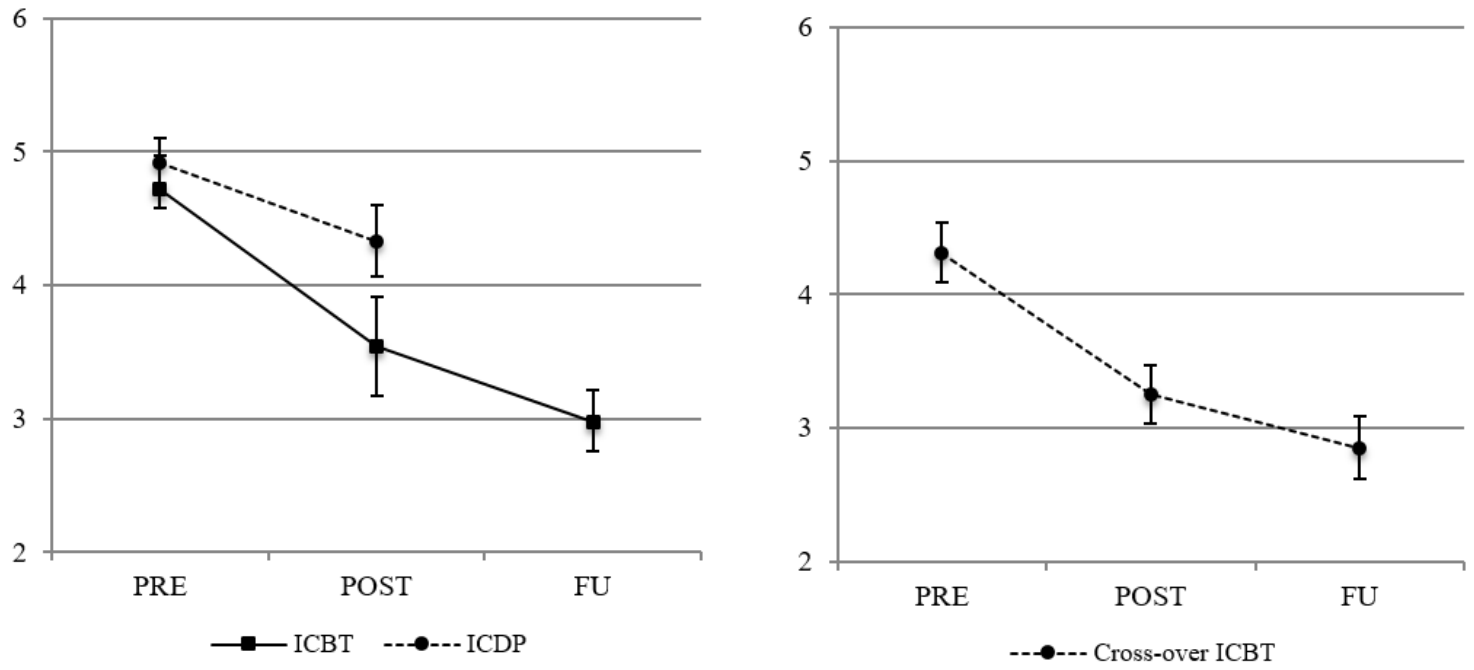

Figure 2: Visual summary of the treatment effects on primary outcome measure

Abbreviations. ICBT=Internet-delivered Cognitive Behaviour Therapy; ICDP=Internet-delivered Child-directed Play.

Note. Based on estimated means derived from the linear mixed model with 95\% confidence intervals; cross-over, and follow-up data based on within-group analysis 

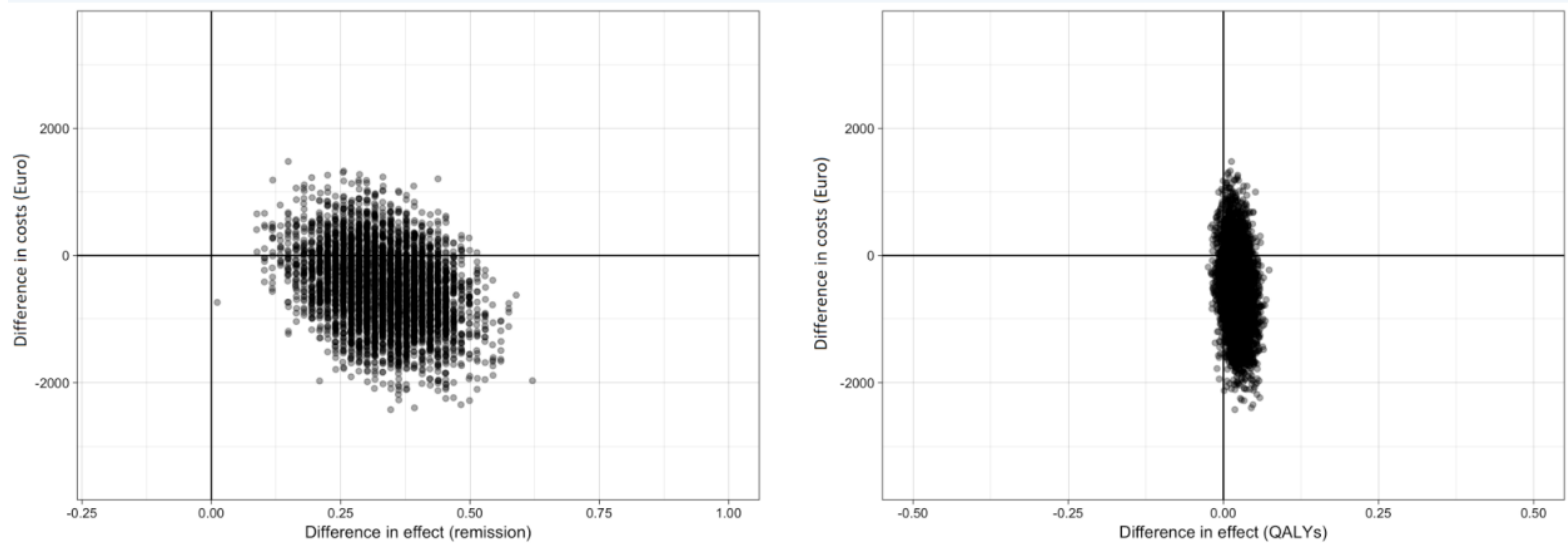

Figure 3: Cost-effectiveness planes in regards to remission (left panel) and QALYs (right panel) Abbreviations. QALYs=Quality-adjusted life years. Note. Remission refers to participant not meeting criteria for principal diagnosis post treatment. 


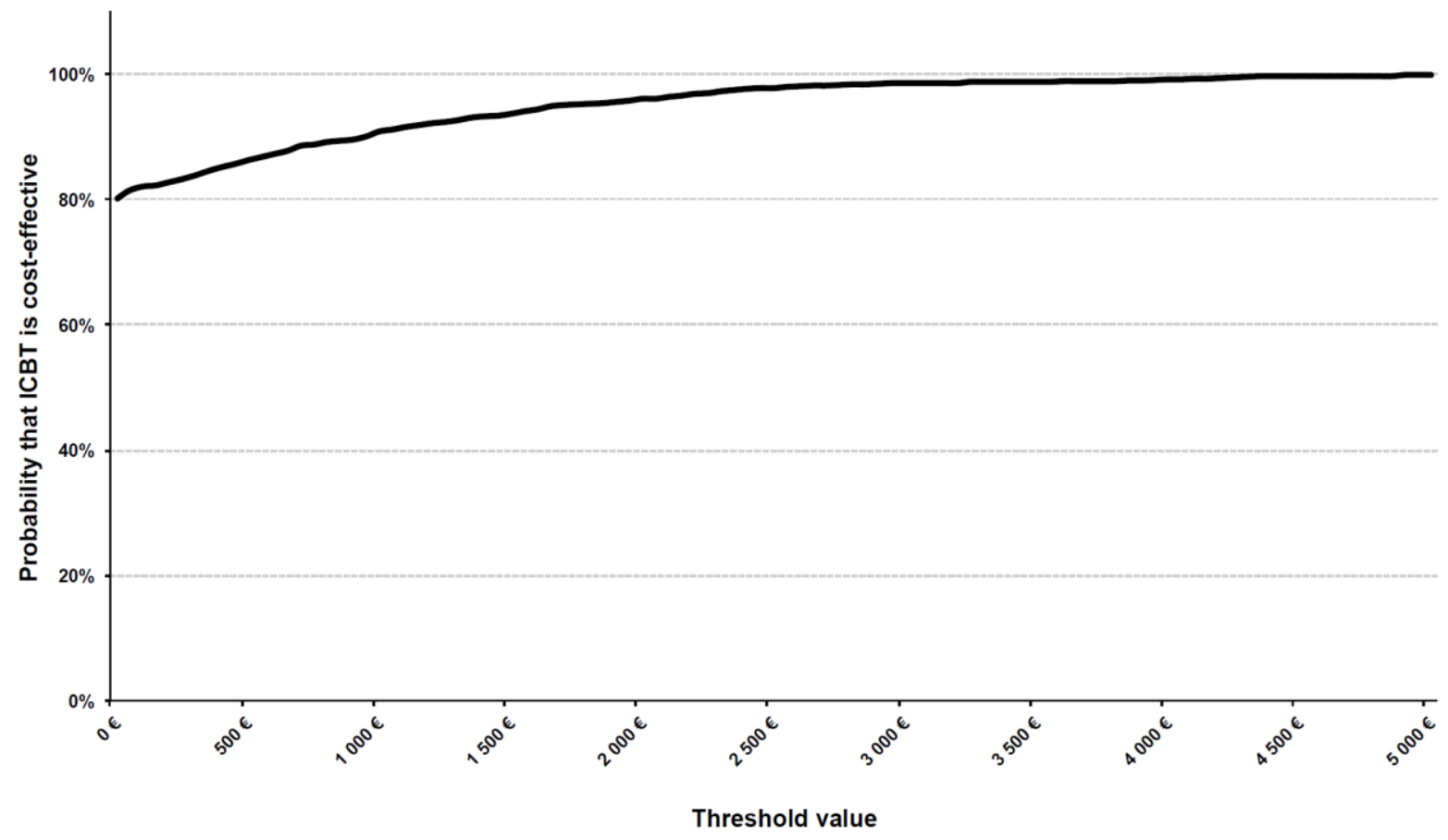

Figure 4: Cost-effectiveness acceptability curve for internet-delivered Cognitive Behaviour Therapy 